\title{
A THEORETICAL EXAMINATION OF DIFFUSIVE MOLECULAR DYNAMICS
}

\author{
G. SIMPSON, M. LUSKIN, AND D.J. SROLOVITZ
}

\begin{abstract}
Diffusive molecular dynamics is a novel model for materials with atomistic resolution that can reach diffusive time scales. The main ideas of diffusive molecular dynamics are to first minimize an approximate variational Gaussian free energy of the system with respect to the mean atomic coordinates (averaging over many vibrational periods), and to then to perform a diffusive step where atoms and vacancies (or two species in a binary alloy) flow on a diffusive time scale via a master equation. We present a mathematical framework for studying this algorithm based upon relative entropy, or Kullback-Leibler divergence. This adds flexibility in how the algorithm is implemented and interpreted. We then compare our formulation, relying on relative entropy and absolute continuity of measures, to existing formulations. The main difference amongst the equations appears in a model for vacancy diffusion, where additional entropic terms appear in our development.
\end{abstract}

\section{INTRODUCTION}

One of the outstanding challenges in atomistic simulation of condensed systems, such as solids, liquids, and glasses, is access to experimentally meaningful length and time scales. The spatial scales amenable to direct molecular dynamics (MD) simulations have grown over time; MD simulations on current large scale parallel computational facilities now reach over $10^{12}$ atoms or approximately $1 \mu \mathrm{m}^{3}$, 7]. Significantly larger length scales can be achieved by application of multiscale modeling techniques that combine atomistic simulations with continuum methods (see, for example, 1, 2, 16, 22, 26, 28, 33). With regard to time, MD simulations have fundamental time scales associated with atomic vibration periods $\left(\sim 10^{-13}\right.$ s), but typical MD time steps are two orders of magnitude smaller. The longest times that have been achieved in large scale MD simulations on special purpose hardware is $\sim 10^{-3} \mathrm{~s}, 30$. More typically MD simulations access times of less than than $10^{-8} \mathrm{~s}$. Hence, reaching laboratory time scales remains amongst the most important challenges in the application of MD today.

Many approaches have been developed to address the molecular dynamics time-scale challenge. Of these, methods based upon transition state theory

Date: September 18, 2018.

GS and ML were supported by US Department of Energy Award de-sc0012733. 
have been widely applied and their continued development remains an active area of research. Transition state theory-based methods rely on rare event ideas in which the system explores a particular energy basin for a long period of time and makes infrequent transitions from one basin to another, $[9,13,27,31,32$. Access to long time scales is provided by replacing the true dynamics within the basin with a stochastic approximation and the prediction of the times between the transitions to other basins. Such methods rely on the transitions being sufficiently rare; i.e., the computational time required to characterize the dynamics within the basin is much shorter than the time between inter-basin transits. The main difficulty with this approach arises when the transitions between basins are not rare; for example, at high temperature or in situations where there are low energy barriers between basins. Another challenge arises in very large systems as the time between rare events occurring somewhere in the system typically scales inversely with system size. Nonetheless, progress has recently been made to extend these methods to large systems, [11, 12, 34.

An important distinction between classes of "events" in many materials science applications is between those that are displacive and those that are diffusive. Displacive events are often collective and involve relative atomic displacements that are small compared with a typical interatomic separation $(\sim 2 \AA)$. Diffusive events, on the other hand, often involve a series of atom or vacancy hops amongst atomic sites. In a solid, the time between hops is commonly many orders of magnitude larger than the atomic vibration period. This type of scale separation is necessary for the application of traditional transition state theory-based approaches. The motion of defects in materials and many types of phase transformations occur through a combination of displacive and diffusive events.

1.1. Diffusive Molecular Dynamics. One method that explicitly takes advantage of this separation in scale between diffusive and displacive events in atomistic simulations is the so-called Diffusive Molecular Dynamics (DMD) method [15, 24]. The idea is to first minimize an approximate free energy (including atomic bonding and atomic vibrational degrees of freedom) of the system with respect to the mean atomic coordinates (averaging over many vibrational periods), and then to perform a diffusive step where atoms and vacancies flow on a diffusive time scale. This free energy is typically described using the variational Gaussian (VG) method [14,18. The time step for such simulations is the relatively long (compared with vibrational) diffusional time scale. In this approach, the search for transition state barriers is replaced by the introduction of an empirical diffusion coefficient or mobility. While this coefficient may be determined directly from atomistic simulation for every local transition, it is commonly viewed as a constant across the entire simulation; this constant can also be determined from purely atomistic 
calculations. This approach is physically sensible when most of the important diffusive events are of the same type, as in an exchange of an atom for a vacancy.

A key element of DMD is that, in contrast to traditional MD, each atomic site has associated with it a continuous probability of occupancy by an atom. See, for example, [19]. We denote this probability $c_{i}$ at atomic site $i$. In the case of a single species (elemental) materials, $c_{i}$ close to zero corresponds to site $i$ being a vacancy with high probability, while $c_{i}$ close to one corresponds to site $i$ being occupied by an atom with high probability. Alloys can also be described in this manner. For example, $c_{i}$ close to zero could correspond to species A while $c_{i}$ close to one could correspond to species B. This can be extended to multiple species, along with vacancies, by the introductions of additional degrees of freedom. Such an extension of the state space, to allow for longer time scale evolution, has also been explored in [29].

1.2. Relative Entropy. One of the tools we make use of in this analysis is relative entropy, or the Kullback-Leibler divergence (KL), 6]. KL is one of the many ways that the distance between two probability measures, such as ensembles, can be computed. It is broadly used in information theory, uncertainty quantification, statistical inverse problems, molecular dynamics, and other applications; see, for instance, $[3,10,17,20,21,25]$ and references therein.

Given two probability measures, $\nu$ and $\mu$ on a common state space, the relative entropy distance between them is given by

$$
\mathcal{R}(\mu \| \nu)= \begin{cases}\mathbb{E}^{\mu}\left[\log \frac{d \mu}{d \nu}\right] & \mu \ll \nu, \\ \infty & \text { otherwise }\end{cases}
$$

Here, $\mu \ll \nu$ if for any measurable set $A$ such that $\nu(A)=0$, we have $\mu(A)=0$ too. In this case, $\mu$ is said to be absolutely continuous with respect to $\nu$.

Relative entropy is a natural tool for this work as it can be directly connected to the Helmholtz free energy of a system. Indeed, the statement that $\mathcal{R} \geq 0$ is equivalent to the Gibbs-Bogoliubov inequality [25]. To motivate this, consider the canonical ensembles associated with potential $V(x)$ and an approximate potential $\hat{V}(x)$ :

$$
\nu(d x)=Z^{-1} e^{-\beta V(x)} d x, \quad \hat{\nu}(d x)=\hat{Z}^{-1} E^{-\beta \hat{V}(x)} d x,
$$

where $Z$ is the partition function, $\beta=\left(k_{\mathrm{B}} T\right)^{-1}, k_{\mathrm{B}}$ is the Boltzmann constant and $T$ is the absolute temperature. Assuming that $\hat{\nu} \ll \nu$,

$$
\mathcal{R}(\hat{\nu} \| \nu)=\beta \mathbb{E}^{\hat{\nu}}[V(x)-\hat{V}(x)]-\log \hat{Z}+\log Z .
$$

Dividing through by $\beta$, and using $\mathcal{R} \geq 0$,

$$
-\beta^{-1} \log Z \leq \beta \mathbb{E}^{\hat{\nu}}[\Delta V]-\beta^{-1} \log \hat{Z},
$$


which is the Gibbs-Bogoliubov inequality. Mathematical treatments of $\mathcal{R}$ and its relationship to other popular measures of distance between probability measures can be found in [6, 8 ].

1.3. Outline. In this work, we formulate DMD using relative entropy, towards the goal of constructing a rigorous mathematical foundation for the algorithm. We also examine under what assumptions the necessary absolute continuity will hold. This framework leads to naturally well posed variational problems that are inherently bounded from below by virtue of $\mathcal{R}$ being non-negative.

Our paper is outlined as follows. In Section 2 we give our formulation of DMD using relative entropy. Then, in Section 3, we compare our expressions with those of the existing formulations of DMD. Several additional explicit calculations are given in the Appendix.

\section{A Relative Entropy Formulation of DMD}

To begin our description of DMD, we work in an extended state space that includes both the positions of the atom sites, $\mathbf{x}_{i} \in \mathbb{R}^{3}$, and the presence/absence of atoms, $a_{i} \in\{0,1\}$. For binary alloys, $a_{i}=1$ could correspond to species $\mathrm{A}$ and $a_{i}=0$ could correspond to species $\mathrm{B}$. In either case, the total number of sites $N$ is fixed and $i=1, \ldots N$. In what follows, we give separate developments of the vacancy and the binary alloy problems.

2.1. DMD Potentials. We assume the existence of a potential $V$, that describes atomic bonding, as in traditional MD. Here, we focus on the class of pair potentials,

$$
V(\mathbf{x})=\sum_{i<j} \phi\left(\left|\mathbf{x}_{i}-\mathbf{x}_{j}\right|\right) .
$$

The associated DMD potential is

$$
V_{\mathrm{DMD}}(\mathbf{x}, \mathbf{a})=\sum_{i<j} a_{i} a_{j} \phi\left(\left|\mathbf{x}_{i}-\mathbf{x}_{j}\right|\right) .
$$

In an elemental material, the potentials $V$ and $V_{\text {DMD }}$ are non-zero only when both atom $i$ and atom $j$ are present (i.e., the interaction between an atom and a vacancy is zero). In the case of a binary alloy (ignoring vacancies), we would have

$$
\begin{aligned}
V_{A B}(\mathbf{x}, \mathbf{a})= & \sum_{i<j} a_{i} a_{j} \phi_{A A}\left(\left|\mathbf{x}_{i}-\mathbf{x}_{j}\right|\right) \\
& +\sum_{i<j}\left[a_{i}\left(1-a_{j}\right)+\left(1-a_{i}\right) a_{j}\right] \phi_{A B}\left(\left|\mathbf{x}_{i}-\mathbf{x}_{j}\right|\right) \\
& +\sum_{i<j}\left(1-a_{i}\right)\left(1-a_{j}\right) \phi_{B B}\left(\left|\mathbf{x}_{i}-\mathbf{x}_{j}\right|\right)
\end{aligned}
$$

where $\phi_{T_{i} T_{j}}$ is the pair potential between a pair of atoms of types $T_{i}$ and $T_{j} \in\{A, B\}$. More sophisticated potentials, such as the embedded atom 
method (EAM), can be used, but for the purpose of this work, it will suffice to consider the pair potential.

\subsection{Free Energy and Relative Entropy for the Vacancy Problem.}

2.2.1. Canonical Ensemble. Letting $D$ be a bounded, open subset of $\mathbb{R}^{3}$, we now formulate the canonical ensemble for a "c $N T$ " (fixed total composition, number of atomic sites and temperature) system, by introducing the distribution

$$
\nu(d \mathbf{x}, \mathbf{a})=Z^{-1} \exp \left\{-\beta\left(V_{\mathrm{DMD}}-\boldsymbol{\mu} \cdot \mathbf{a}\right)\right\} d \mathbf{x},
$$

with $\boldsymbol{\mu}=\left(\mu_{1}, \ldots, \mu_{N}\right)$ is a set of chemical potentials. Chemical potential $\mu_{i}$ is associated with site $i$; each site has its own reservoir. While this is in the form of a generalized grand canonical ensemble, we view the chemical potentials as Lagrange multipliers enforcing the constraints

$$
\mathbb{E}^{\nu}\left[a_{i}\right]=c_{i}, \quad i=1, \ldots N .
$$

It is in this way that our $\nu$ is akin to a canonical ensemble, with the mean occupancy values, the c, fixed.

The partition function is

$$
Z=\sum_{\mathbf{a}} \int_{D} \exp \left\{-\beta\left(V_{\mathrm{DMD}}(\mathbf{x}, \mathbf{a})-\boldsymbol{\mu} \cdot \mathbf{a}\right)\right\} d \mathbf{x},
$$

and the ensemble averages require integration in space over $D$ along with summation over all possible configurations of $\mathbf{a}=\left(a_{1}, \ldots, a_{N}\right)$. We thus assume:

Assumption 1. For potential $V$, bounded set $D$, and occupancies $\mathbf{c}$ with $c_{i} \in(0,1)$, the chemical potentials $\mu_{i}$ are finite and the partition function is finite and positive.

We describe the distribution (2.4) as the "true" DMD distribution. The associated free energy is

$$
F=-\beta^{-1} \log Z+\boldsymbol{\mu} \cdot \mathbf{c} .
$$

In $\mathrm{DMD}$, the concentrations are allowed to evolve under

$$
\dot{c}_{i}=\sum_{j \in N(i)} k_{i j}\left(\frac{\partial F}{\partial c_{j}}-\frac{\partial F}{\partial c_{i}}\right)
$$

where $k_{i j}$ is a mobility term, and $N(i)$ includes neighbors of site $i$; both are, a priori, unconstrained. We note that (2.8) is not the evolution studied by Sarkar [23, due to the challenge of computing the partition function; rather, they employ an approximate free energy. Nevertheless, we contend that, were computational complexity not an obstacle, (2.8) is the desired form of dynamics. 
As the $c_{i}$ evolve, the equilibrium positions of the atomic sites will also change, and can be obtained by computing

$$
\mathbb{E}^{\nu}\left[\mathbf{x}_{i}\right]
$$

2.2.2. Approximate Potential. Due to the computational challenge in evaluating the partition function, (2.6), an approximate potential is introduced, $\hat{V}$, with a corresponding probability distribution, $\hat{\nu}$. This potential is then tuned to provide a "best" approximation of $\nu$ by $\hat{\nu}$. Here, we consider the following quadratic (harmonic) potential in the case of an elemental material (including vacancies)

$$
\hat{V}_{\mathrm{DMD}}(\mathbf{x}, \mathbf{a} ; \mathbf{k}, \mathbf{X})=\sum_{i} \frac{a_{i} k_{i}}{2}\left|\mathbf{x}_{i}-\mathbf{X}_{i}\right|^{2} .
$$

$\mathbf{X}$ and $\mathbf{k}$ are parameters approximating the mean atomic position and its characteristic fluctuation. The approximate probability distribution is now

$$
\hat{\nu}(d \mathbf{x}, \mathbf{a} ; \mathbf{k}, \mathbf{X})=\hat{Z}^{-1} \exp \left\{-\beta\left(\hat{V}_{\mathrm{DMD}}-\hat{\boldsymbol{\mu}} \cdot \mathbf{a}\right)\right\} d \mathbf{x} .
$$

Again, the $\hat{\mu}_{i}$ are Lagrange multipliers which must satisfy the analog of (2.5),

$$
\mathbb{E}^{\hat{\nu}}\left[a_{i}\right]=c_{i} .
$$

For this case, we can provide expressions for both the partition function and the $\hat{\mu}_{i}$. The approximate partition function is

$$
\hat{Z}=\Pi_{i=1}^{N}\left(|D|+e^{\beta \hat{\mu}_{i}} Z_{i}\right), \quad Z_{i} \equiv \int_{D} \exp \left\{-\frac{\beta k_{i}}{2}\left|\mathbf{x}_{i}-\mathbf{X}_{i}\right|^{2}\right\} d \mathbf{x}_{i},
$$

where $|D|$ is the volume of the computational domain. The chemical potentials $\hat{\mu}_{i}$, defined by (2.12), satisfy (see (A.2))

$$
\mathbb{E}^{\hat{\nu}}\left[a_{i}\right]=\frac{e^{\beta \hat{\mu}_{i}} Z_{i}}{|D|+e^{\beta \hat{\mu}_{i}} Z_{i}}=c_{i} .
$$

These can then be solved for each $i$ to obtain:

$$
\hat{\mu}_{i}=\beta^{-1} \log \left(\frac{c_{i}}{1-c_{i}} \cdot \frac{|D|}{Z_{i}}\right) .
$$

The expression for the chemical potentials, (2.15), can also be used to write a simplified expression for the partition function:

$$
\hat{Z}=|D|^{N} \Pi_{i=1}^{N} \frac{1}{1-c_{i}} .
$$

The approximate free energy is now given by

$$
\begin{array}{r}
-\beta^{-1} \log \hat{Z}+\hat{\boldsymbol{\mu}} \cdot \mathbf{c}=\beta^{-1} \sum_{i=1}^{N} c_{i} \log c_{i}+\left(1-c_{i}\right) \log \left(1-c_{i}\right) \\
+\left(c_{i}-1\right) \log |D|-c_{i} \log Z_{i} .
\end{array}
$$


Note that because $D$ is a bounded set, $\mathbf{X}_{i}$ is not exactly the mean position of atomic site $i$. See Appendix $\mathrm{A}$ for details.

2.2.3. Relative Entropy and the Generalized Variational Approach. The basis for optimizing (2.10) so that $\hat{\nu}$ provides the best match to $\nu$ is inspired by the Gibbs-Bogoliubov inequality and the variational Gaussian approach [14,18]. As alluded to in the introduction, this is closely related to the relative entropy metric.

Let us assume that $V$, the primitive potential in this problem, is bounded on the set $D 1$ In particular, we assume

Assumption 2. There exists a constant $C>0$ such that for all $\mathbf{x} \in D^{N}$ and for all $\mathbf{a}$,

$$
\left|V_{\mathrm{DMD}}(\mathbf{x}, \mathbf{a})\right| \leq C
$$

Under these assumptions, we have the necessary absolute continuity to proceed with using $\mathcal{R}$ :

Proposition 1. Subject to Assumptions 1 and [2, $\hat{\nu} \ll \nu$ with RadonNikodym derivative

$$
\frac{d \hat{\nu}}{d \nu}(\mathbf{x}, \mathbf{a})=\frac{Z}{\hat{Z}} \exp \left\{\beta\left(V_{\mathrm{DMD}}-\hat{V}_{\mathrm{DMD}}+\beta(\hat{\boldsymbol{\mu}}-\boldsymbol{\mu}) \cdot \mathbf{a}\right\}\right.
$$

and

$$
\mathcal{R}(\hat{\nu} \| \nu)=\beta \mathbb{E}^{\hat{\nu}}[\Delta V]+\beta(\hat{\boldsymbol{\mu}}-\boldsymbol{\mu}) \cdot \mathbb{E}^{\hat{\nu}}[\mathbf{a}]+\log Z-\log \hat{Z}<\infty .
$$

Proof. Let $A$ be any measurable subset of the state space, $(\{0,1\} \times D)^{N}$, of the form

$$
A=\Pi_{i=1}^{N}\left(a_{i} \times B_{i}\right)
$$

where each $B_{i}$ is a Lebesgue measurable subset of $D \subset \mathbb{R}^{d}$. If $\nu(A)=0$, then

$$
0=\int_{B_{1} \times B_{2} \ldots \times B_{N}} \exp \left\{-\beta\left(V_{\mathrm{DMD}}(\mathbf{x}, \mathbf{a})-\boldsymbol{\mu} \cdot \mathbf{a}\right)\right\} d \mathbf{x} \geq e^{-\beta C-\beta \sum_{i}\left|\mu_{i}\right|} \Pi_{i}\left|B_{i}\right|,
$$

where $\left|B_{i}\right|$ is the Lebesgue measure of the set $B_{i}$ and $C$ is the constant from Assumption 2. Since the $\mu_{i}$ are assumed to be finite, we can thus infer that the only way $\nu(A)=0$ is if at least one of the $B_{i}$ has Lebesgue measure zero, regardless of the particular site occupancy values, $a_{i}$. Then, since the $k_{i}$ defining $\hat{V}_{\mathrm{DMD}}$ are finite,

$$
\begin{aligned}
\hat{\nu}(A) & =\hat{Z}^{-1} \int_{B_{1} \times B_{2} \ldots \times B_{N}} \exp \left\{-\beta\left(\hat{V}_{\mathrm{DMD}}(\mathbf{x}, \mathbf{a})-\hat{\boldsymbol{\mu}} \cdot \mathbf{a}\right)\right\} d \mathbf{x} \\
& \leq \hat{Z}^{-1} e^{\beta \sum_{i}\left|\hat{\mu}_{i}\right|} \Pi_{i}\left|B_{i}\right|=0 .
\end{aligned}
$$

\footnotetext{
${ }^{1}$ While this formally excludes potentials which diverge near the origin, such as the Lennard-Jones potential, such divergences are routinely avoided by choosing a cut-off at an appropriately small interatomic separation.
} 
Since this holds for all sets of type (2.21), we conclude it that for all measurable subsets of $(\{0,1\} \times D)^{N}$ for which $\nu(A)=0$ we must have $\hat{\nu}(A)=0$. This gives us absolute continuity of the measures.

The Radon-Nikodym derivative is then given by (2.19) and $\mathcal{R}$ is given by (2.20). By our assumptions, $\mathcal{R}$ will be finite.

Since $\mathcal{R} \geq 0$, we can use (2.12) and (2.17) to express (2.20) as

$$
\begin{gathered}
F \leq E^{\hat{\nu}}[\Delta V]+\beta^{-1} \sum_{i=1}^{N} c_{i} \log c_{i}+\left(1-c_{i}\right) \log \left(1-c_{i}\right)-c_{i} \log Z_{i} \\
+\left(c_{i}-1\right) \log |D| .
\end{gathered}
$$

We thus define the approximate DMD free energy, which is an upper bound on the true DMD free energy, as

$$
\begin{gathered}
\hat{F} \equiv E^{\hat{\nu}}[\Delta V]+\beta^{-1} \sum_{i=1}^{N} c_{i} \log c_{i}+\left(1-c_{i}\right) \log \left(1-c_{i}\right)-c_{i} \log Z_{i} \\
+\left(c_{i}-1\right) \log |D| .
\end{gathered}
$$

The DMD algorithm for the vacancy problem proceeds in two steps:

(1) Find a minimizer of $\hat{F}$ over $\mathbf{k}$ and $\mathbf{X}$, with $\mathbf{X}_{i} \in D$ and $k_{i} \geq 0$.

(2) Approximate the dynamics of (2.8), substituting $\hat{F}$ for $F$.

Thus, since the Gibbs-Bogliubov inequality is equivalent to the statement that the relative entropy is non-negative, the first step in the above algorithm is to find the best approximation, with respect to relative entropy, of $\nu$ over a class of distributions of type $\hat{\nu}$.

2.3. Free Energy and Relative Entropy for a Binary Mixture. Mirroring our examination of the vacancy problem, we consider the analogous formulation for a binary alloy.

2.3.1. Canonical Ensemble. For a binary mixture, many of the calculations are similar, or even simpler, than for the case of an elemental material with vacancies. First, we formulate the $\nu_{\mathrm{AB}}$ distribution:

$$
\nu_{\mathrm{AB}}(d \mathbf{x}, \mathbf{a})=Z_{\mathrm{AB}}^{-1} \exp \left\{-\beta\left(V_{\mathrm{AB}}-\boldsymbol{\mu} \cdot \mathbf{a}\right)\right\} d \mathbf{x} .
$$

We continue to assume Assumption 1 holds, adapted to the binary mixture case.

2.3.2. Approximate Ensemble. Now, instead of the approximate potential given by (2.10) for the vacancy problem, we assume

$$
\hat{V}_{\mathrm{AB}}=\sum_{i} \frac{k_{i}}{2}\left|\mathbf{x}_{i}-\mathbf{X}_{i}\right|^{2} .
$$

The distinction here is that because each site always contains an atom of species $\mathrm{A}$ or $\mathrm{B}$, the potential is always non-zero. In contrast, the potential 
associated with a vacancy is always zero and a vacancy is never subject to a force. Proceeding with the potential (2.26), we find

$$
\hat{\nu}_{\mathrm{AB}}(d \mathbf{x}, \mathbf{a} ; \mathbf{k}, \mathbf{X})=\hat{Z}_{\mathrm{AB}}^{-1} \exp \left\{-\beta\left(\hat{V}_{\mathrm{AB}}-\hat{\boldsymbol{\mu}} \cdot \mathbf{a}\right)\right\} d \mathbf{x},
$$

where the chemical potentials are chosen to satisfy $\mathbb{E}^{\hat{\nu} \mathrm{AB}}\left[a_{i}\right]=c_{i}$. In this case

$$
\hat{Z}_{\mathrm{AB}}=\Pi_{i=1}^{N}\left(1+e^{\beta \hat{\mu}_{i}}\right) Z_{i},
$$

where $Z_{i}$ defined as in (2.13). Hence, we can immediately write the chemical potential as

$$
\hat{\mu}_{i}=\beta^{-1} \log \left(\frac{c_{i}}{1-c_{i}}\right) .
$$

The free energy is then given by

$$
-\beta^{-1} \log \hat{Z}+\boldsymbol{\mu} \cdot \mathbf{c}=\beta^{-1} \sum_{i=1}^{N} c_{i} \log c_{i}+\left(1-c_{i}\right) \log \left(1-c_{i}\right)-\log Z_{i}
$$

2.3.3. Relative Entropy for the Binary Mixture. We next consider the relative entropy minimization problem for the binary mixture, requiring $\hat{\nu}_{\mathrm{AB}} \ll$ $\nu_{\mathrm{AB}}$. Using the same approach as in the vacancy case, we will also assume the boundedness of $V_{\mathrm{AB}}$, in the same spirit as Assumption 2.

Proposition 2. Under Assumptions 1 and 2 for the binary mixture case, $\hat{\nu}_{\mathrm{AB}} \ll \nu_{\mathrm{AB}}$ with the associated Radon-Nikodym derivative, and

$$
\mathcal{R}\left(\hat{\nu}_{\mathrm{AB}} \| \nu_{\mathrm{AB}}\right)=\beta \mathbb{E}^{\hat{\nu}_{\mathrm{AB}}}[\Delta V]+\beta(\hat{\boldsymbol{\mu}}-\boldsymbol{\mu}) \cdot \mathbf{c}-\log \hat{Z}_{\mathrm{AB}}+\log Z_{\mathrm{AB}}<\infty .
$$

As before, we can reformulate this as a free energy statement,

$$
\begin{aligned}
& \underbrace{-\beta^{-1} \log Z_{\mathrm{AB}}+\boldsymbol{\mu} \cdot \mathbf{c}}_{F_{\mathrm{AB}}} \\
& \leq \underbrace{\mathbb{E}^{\hat{\nu}_{\mathrm{AB}}}[\Delta V]+\beta^{-1} \sum_{i=1}^{N} c_{i} \log c_{i}+\left(1-c_{i}\right) \log \left(1-c_{i}\right)-\log Z_{i}}_{\hat{F}_{\mathrm{AB}}} .
\end{aligned}
$$

The simulation now proceeds as above, with a minimizer $\mathcal{R}$ over $\mathbf{k}$ and $\mathbf{X}$ while the $c_{i}$ evolve.

\section{Discussion}

\subsection{Relation to Existing DMD Formulations.}


3.1.1. Vacancy Problem. We compare (2.24) to the DMD free energy for the vacancy problem found in [15, 23, 24]. Indeed, if one rewrites equation (3) from the original 2011 DMD paper [15] for the case of the pair potential (in the classical approximation - i.e., for Planck's constant equals zero), the expression is:

$$
\begin{aligned}
F_{2011}= & \sum_{i<j} c_{i} c_{j}\left(\frac{2 \pi}{\beta k_{i}}\right)^{\frac{d}{2}}\left(\frac{2 \pi}{\beta k_{j}}\right)^{\frac{d}{2}} \iint_{\mathbb{R}^{2 d}} \phi\left(\left|\mathbf{x}_{i}-\mathbf{x}_{j}\right|\right) e^{-\frac{\beta k_{i}}{2}\left|\mathbf{x}_{i}-\mathbf{x}_{i}\right|^{2}} e^{-\frac{\beta k_{i}}{2}\left|\mathbf{x}_{j}-\mathbf{x}_{j}\right|^{2}} d \mathbf{x}_{i} d \mathbf{x}_{j} \\
& +\beta^{-1} \sum_{i=1}^{N} \frac{d}{2} c_{i}\left(\log \frac{\beta k_{i}}{2 \pi}-1\right)+c_{i} \log c_{i}+\left(1-c_{i}\right) \log \left(1-c_{i}\right) .
\end{aligned}
$$

Some of the differences between (3.1) and (2.24) can be reconciled by the use of a mean field approximation, by which (2.2) is replaced with

$$
V_{\mathrm{DMD}, \mathrm{mf}}=\sum_{i<j} c_{i} c_{j} \phi\left(\left|\mathbf{x}_{i}-\mathbf{x}_{j}\right|\right)
$$

where the $c_{i}$ take continuous values between 0 and 1 . This approximation simplifies some of the computations. For instance, the $\mu_{i}$ are now explicit:

$$
\mu_{i}=\beta^{-1} \log \left(\frac{c_{i}}{1-c_{i}}\right)
$$

and the numerical estimation of $\mathbb{E}^{\hat{\nu}}\left[V_{\mathrm{DMD}}\right]$ is simplified. Notice that (3.3) is the same quantity as we obtained in our examination of the binary mixture, (2.29). This will hold generically when the potential for our distribution, whether true or approximate, does not explicitly depend on a. Furthermore, in the mean field case, the finiteness of the partition function, assumed in Assumption 1, is implied by boundedness of the mean field potential over the set $D$ for all $\mathbf{c} \in[0,1]^{N}$; thus, an assumption like Assumption 2 is required.

However, the mean field approximation does not fully account for the differences. Part of the discrepancy may be attributed to the choice of the state space; Sarkar et al. [15, 24] use $\left(\{0,1\} \times \mathbb{R}^{d}\right)^{N}$. Formally, as $D \rightarrow \mathbb{R}^{d}$,

$$
Z_{i} \rightarrow\left(\frac{2 \pi}{\beta k_{i}}\right)^{\frac{d}{2}}, \quad \mathbb{E}^{\hat{\nu}}\left[\hat{V}_{\mathrm{DMD}}\right] \rightarrow \beta^{-1} \frac{d}{2} \sum_{j=1}^{N} c_{j},
$$

and (2.24) tends to

$$
\hat{F} \rightarrow F_{2011}+\beta^{-1} \sum_{i=1}^{N}\left(c_{i}-1\right) \log |D|,
$$

where we have not taken $|D|$ to the limit in the last expression. This dependence on $|D|$ is a finite size correction to the free energy. As $D \rightarrow \mathbb{R}^{d}$, this becomes unbounded. However, this term does not alter the algorithm since we are only concerned with differences of free energies rather than absolute magnitudes. Indeed, during the first part of the algorithm, where a local minimizer of $\hat{F}$ is sought over $\mathbf{k}$ and $\mathbf{X}$ with fixed $\mathbf{c}$, there are no contributions $\propto \log |D|$. And in the second part of the algorithm, under the 
dynamics of type (2.8), where $\hat{F}$ is used in place of $F$, the $\log |D|$ terms cancel one another.

The $\log |D|$ terms also cancel under the more sophisticated dynamics used in [4, 15, 24]. There, the dynamics are given by the master equation

$$
\dot{c}_{i}=\sum_{j \in N(i)} \nu e^{-\beta Q_{\mathrm{m}}}\left\{c_{j}\left(1-c_{i}\right) e^{-\beta\left(f_{i}-f_{j}\right)}-c_{i}\left(1-c_{j}\right) e^{-\beta\left(f_{j}-f_{i}\right)}\right\},
$$

with

$$
f_{i} \equiv \frac{\partial \hat{F}}{\partial c_{i}}-\beta^{-1} \log \left(\frac{c_{i}}{1-c_{i}}\right) .
$$

Because of the differentiation with respect to $c_{i}$ in the previous expression, the $\log |D|$ term does not appear in the differences, $f_{i}-f_{j}$.

A more substantive difference between the existing DMD literature and our analysis is the notion of the ensemble averaged site positions. As derived in the Appendix (A.3),

$$
\begin{aligned}
\mathbb{E}^{\hat{\nu}}\left[\mathbf{x}_{i}\right] & =\frac{\int_{D} \mathbf{x}_{i} d \mathbf{x}_{i}+e^{\beta \hat{\mu}_{i}} \int_{D} \mathbf{x}_{i} \exp \left\{-\frac{\beta k_{i}}{2}\left|\mathbf{x}_{i}-\mathbf{X}_{i}\right|^{2}\right\} d \mathbf{x}_{i}}{|D|+e^{\beta \hat{\mu}_{i}} \int_{D} \exp \left\{-\frac{\beta k_{i}}{2}\left|\mathbf{x}_{i}-\mathbf{X}_{i}\right|^{2}\right\} d \mathbf{x}_{i}} \\
& =\left(1-c_{i}\right) \frac{\int_{D} \mathbf{x}_{i} d \mathbf{x}_{i}}{|D|}+c_{i} \frac{\int_{D} \mathbf{x}_{i} \exp \left\{-\frac{\beta k_{i}}{2}\left|\mathbf{x}_{i}-\mathbf{X}_{i}\right|^{2}\right\} d \mathbf{x}_{i}}{Z_{i}}
\end{aligned}
$$

where we have used (2.15) to simplify the expression. For simulations in computational domains that are symmetric about the origin, such as $D=$ $(-L, L)^{d}$ or a hypersphere, the first term in this expression vanishes and $\mathbb{E}^{\hat{\nu}}\left[\mathbf{x}_{i}\right] \rightarrow c_{i} \mathbf{X}_{i}$.

An alternative notion of mean position could be useful in this case. Consider:

$$
\frac{\mathbb{E}^{\hat{\nu}}\left[a_{i} \mathbf{x}_{i}\right]}{\mathbb{E}^{\hat{\nu}}\left[a_{i}\right]}=\frac{1}{Z_{i}} \int_{D} \mathbf{x}_{i} \exp \left\{-\frac{\beta k_{i}}{2}\left|\mathbf{x}_{i}-\mathbf{X}_{i}\right|^{2}\right\} d \mathbf{x}_{i},
$$

where we have made use of (A.4). Now, as $D$ tends to $\mathbb{R}^{d}$, (3.9) recovers $\mathbf{X}_{i}$. This weighted averaging is inspired by the mathematical theory of multiphase flow (see, for instance, [5]).

Working with $\mathbb{R}^{d}$ is inherently problematic, as the measures defined as in (2.4) and (2.11) do not lead to well defined probability measures, even under the mean field approximation. Consider, for example, $\hat{\nu}$ with $N=1$. For this problem the partition function would be

$$
\sum_{a_{1}=0}^{1} \int_{\mathbb{R}^{d}} \exp \left\{-\beta \frac{a_{1} k_{1}}{2}\left|\mathbf{x}_{i}-\mathbf{X}_{i}\right|^{2}+\beta \hat{\mu}_{1} a_{1}\right\} d \mathbf{x}_{1}=\infty
$$

since, in the case $a_{1}=0$, we are integrating $d \mathbf{x}_{1}$ over the whole space.

However, we contend that while (3.1) may give rise to physically consistent simulations, it is not based on a variational principle, and instead, 
practitioners should use (2.11). In order to make use of $\mathcal{R}$ and arrive at a variational formulation, it is essential that the distributions be well defined probability distributions, with finite partition functions and that absolute continuity holds. We note that this is not an artifact of our mathematical analysis based on relative entropy. As the Gibbs-Bogliubov inequality is a restatement of the non-negativity of relative entropy, it has the same underlying assumptions of absolute continuity and well defined measures.

One way to correct (3.8) is to alter the choice of (2.10). Suppose we mimic what is done in the binary mixture (which does not suffer from these problems), and took

$$
\hat{V}_{\mathrm{DMD}}=\sum_{i} \frac{k_{i}}{2}\left|\mathbf{x}_{i}-\mathbf{X}_{i}\right|^{2}
$$

We would replicate (2.28) for the partition function and, with this revised value, the free energy would be

$$
\hat{F}_{\mathrm{DMD}}=\mathbb{E}^{\hat{\nu}}[\Delta V]+\beta^{-1} \sum_{i=1}^{N} c_{i} \log c_{i}+\left(1-c_{i}\right) \log \left(1-c_{i}\right)-\log Z_{i} .
$$

Now, as $D \rightarrow \mathbb{R}^{d}$, we obtain

$$
\begin{gathered}
\hat{F}_{\mathrm{DMD}} \approx \mathbb{E}^{\hat{\nu}}\left[V_{\mathrm{DMD}}\right]+\beta^{-1} \sum_{i=1}^{N} c_{i} \log c_{i}+\left(1-c_{i}\right) \log \left(1-c_{i}\right) \\
+\frac{d}{2}\left(\log \frac{\beta k_{i}}{2 \pi}-1\right) .
\end{gathered}
$$

This formulation has the advantage that $\mathbb{E}^{\hat{\nu}}\left[\mathbf{x}_{i}\right] \rightarrow \mathbf{X}_{i}$ as $D \rightarrow \mathbb{R}^{d}$. However, notice now that there is no $c_{i}$ multiplying the last expression in (3.13), as in (3.1).

3.1.2. Binary Mixture Problem. As noted, if we make a mean field approximation, then as $D \rightarrow \mathbb{R}^{d}$, we recover the expression found in [4], which we do not reproduce here.

3.2. Advantages of Relative Entropy. One of the main advantages of the formulation given here is that the question of relative entropy minimization is a rigorously defined variational problem, forcing us to confront problems such as that associated with domain size. Indeed, for DMD we have

Theorem 1. Given $K \in(0, \infty)$ and the open bounded subset $D$ of $\mathbb{R}^{d}$, let

$$
\mathcal{A}_{D, K}=\left\{\gamma \text { of the form (2.11) } \mid \mathbf{X}_{i} \in \bar{D}, \quad k_{i} \in[0, K]\right\} .
$$

Then if $\left\{\gamma_{n}\right\}$ is a minimizing sequence of $\mathcal{R}(\cdot \| \nu)$, it has a subsequential limit such that

$$
\lim _{n \rightarrow \infty} \mathcal{R}\left(\gamma_{n} \| \nu\right)=\mathcal{R}\left(\gamma_{\star} \| \nu\right)=\inf _{\gamma \in \mathcal{A}} \mathcal{R}(\gamma \| \nu)
$$


and

$$
\gamma_{n} \stackrel{\mathrm{TV}}{\rightarrow} \gamma_{\star}
$$

Note that by our previous assumptions on $V$ and $\boldsymbol{\mu}$, that they are bounded on $D$ for $c_{i} \in(0,1)$, we are assured that the elements of $\mathcal{A}_{D, K}$ are absolutely continuous with respect to such a $\nu$, and have finite $D_{k l}$.

Proof. We claim $\mathcal{A}_{D, K}$ is weakly compact; see Appendix B. Therefore, any sequence has a weak limit, $\gamma_{n_{k}} \stackrel{\mathrm{W}}{\rightarrow} \gamma_{\star}$. Then, by the lower semicontinuity of $\mathcal{R}(\cdot \| \nu)$ (Proposition 2.1 of [20]) we have (3.15), then we can infer (3.16) (Lemma 2.4 of [20]).

Thus, the free energy minimization part of the algorithm is well posed in the sense that if we take a minimizing sequence it has a subsequential limit.

Another advantage of this formulation of the problem is that it allows one to consider more general parameterizations of the synthetic distribution, $\hat{\nu}$. Indeed, one could imagine any distribution parameterized by some collection of variables, denoted collectively by $\mathbf{p}$, and proceed as above; first, one finds a local minimizer of $\mathcal{R}\left(\hat{\nu}_{\mathbf{p}} \| \nu\right)$. Using that, the free energy gradients with respect to $\mathbf{c}$ are computed at this stationary point, and this drives the dynamic evolution of the $c_{i}$ 's. Provided this admissible class is closed (in some sense), we are ensured that its minimization problem is well posed too.

3.3. Open Problems and Future Work. Here, we have presented a mathematical framework for DMD, and we have given particular attention to the question of free energy minimization. One point we have not addressed is the temporal evolution problem, and how the master equation will be influenced by the use of the approximate free energy in place of the true DMD free energy. Indeed, we note that while the minimization of free energy ensures that $\hat{\nu}$ is close to $\nu$ in the sense of relative entropy (and hence, total variation), we cannot conclude that

$$
\left|\frac{\partial F}{\partial c_{i}}-\frac{\partial \hat{F}}{\partial c_{i}}\right|,
$$

errors in the dynamics of both (2.8) and (3.6) are small. As of now, this remains an unconstrained approximation that merits investigation. It can be shown that

$$
\frac{\partial F}{\partial c_{i}}=\mu_{i}
$$

and, at a minimizer of $\mathcal{R}$,

$$
\frac{\partial \hat{F}}{\partial c_{i}}=\hat{\mu}_{i}+\beta \operatorname{Cov}_{\hat{\nu}}\left(\Delta V, \partial_{c_{i}} \hat{\boldsymbol{\mu}} \cdot \mathbf{a}\right) .
$$

Calculations of these can be found in the appendix. Similar expressions hold under the mean field approximation. 
The validity of the mean field approximation used in [4, 15, 24] remains to be explored. As noted, this dramatically simplifies the calculations and immediately gives the "true" chemical potentials (3.3). The solvability in the general case is also an open problem.

Finally, there is the question of how, or in what sense, does DMD, as a model, approximate any specific, more primitive MD model, such as Langevin dynamics. More specifically, since DMD removes the stochastic nature of primitive MD models, replacing the displacive dynamics with a finite-temperature, energy minimization, DMD is deterministic. Similarly, the stochastic or random walk-like diffusive dynamics in primitive MD becomes deterministic in DMD. The question of when this is and is not acceptable remains to be explored.

\section{Appendix A. Detailed Calculations}

A.1. Partition Functions. To obtain the approximate partition function (2.13), we employ the following procedure:

$$
\begin{aligned}
\hat{Z} & =\sum_{\mathbf{a}} \int_{D^{N}} \exp \{-\beta(\hat{V}(\mathbf{x}, \mathbf{a} ; \mathbf{k}, \mathbf{X})-\hat{\boldsymbol{\mu}} \cdot \mathbf{a})\} d \mathbf{x} \\
& =\Pi_{i=1}^{N}\left\{\sum_{a_{i}} \int_{D} \exp \left\{-\frac{\beta a_{i} k_{i}}{2}\left|\mathbf{x}_{i}-\mathbf{X}_{i}\right|^{2}+\beta \hat{\mu}_{i} a_{i}\right\} d \mathbf{x}_{i}\right\} \\
& =\Pi_{i=1}^{N}\left\{|D|+e^{\hat{\mu}_{i}} \int_{D} \exp \left\{-\frac{\beta k_{i}}{2}\left|\mathbf{x}_{i}-\mathbf{X}_{i}\right|^{2}\right\} d \mathbf{x}_{i}\right\} \\
& =\Pi_{i=1}^{N}\left\{|D|+e^{\hat{\mu}_{i}} Z_{i}\right\} .
\end{aligned}
$$

A.2. Chemical Potentials. To obtain (2.15), we apply (A.1) to obtain

$$
\begin{aligned}
\mathbb{E}^{\hat{\nu}}\left[a_{i}\right] & =\frac{\Pi_{j \neq i}^{N}\left\{|D|+e^{\beta \hat{\mu}_{j}} Z_{i}\right\}}{\hat{Z}}\left\{\sum_{a_{i}} \int_{D} a_{i} \exp \left\{-\frac{\beta a_{i} k_{i}}{2}\left|\mathbf{x}_{i}-\mathbf{X}_{i}\right|^{2}+\beta \hat{\mu}_{i} a_{i}\right\} d \mathbf{x}_{i}\right\} \\
& =\frac{e^{\beta \hat{\mu}_{i}} Z_{i}}{|D|+e^{\beta \hat{\mu}_{i}} Z_{i}} .
\end{aligned}
$$

Since $\mathbb{E}^{\hat{\nu}}\left[a_{i}\right]=c_{i}$, we solve for $\hat{\mu}_{i}$ in terms of $c_{i}$.

A.3. Mean Position. The expectation value of the atomic position $\mathbf{x}_{i}$ is

$$
\begin{aligned}
\mathbb{E}^{\hat{\nu}}\left[\mathbf{x}_{i}\right] & =\frac{\Pi_{j \neq i}^{N}\left\{|D|+e^{\beta \hat{\mu}_{j}} Z_{i}\right\}}{\hat{Z}}\left\{\sum_{a_{i}} \int_{D} \mathbf{x}_{i} \exp \left\{-\frac{\beta a_{i} k_{i}}{2}\left|\mathbf{x}_{i}-\mathbf{X}_{i}\right|^{2}+\beta \hat{\mu}_{i} a_{i}\right\} d \mathbf{x}_{i}\right\} \\
& =\frac{1}{|D|+e^{\beta \hat{\mu}_{i}} Z_{i}}\left(\int_{D} \mathbf{x}_{i} d \mathbf{x}_{i}+e^{\beta \hat{\mu}_{i}} \int_{D} \mathbf{x}_{i} \exp \left\{-\frac{\beta k_{i}}{2}\left|\mathbf{x}_{i}-\mathbf{X}_{i}\right|^{2}\right\} d \mathbf{x}_{i}\right)
\end{aligned}
$$


and the weighted mean position is given by

$$
\begin{aligned}
\mathbb{E}^{\hat{\nu}}\left[a_{i} \mathbf{x}_{i}\right] & =\frac{\Pi_{j \neq i}^{N}\left\{|D|+e^{\beta \hat{\mu}_{j}} Z_{i}\right\}}{\hat{Z}}\left\{\sum_{a_{i}} \int_{D} a_{i} \mathbf{x}_{i} \exp \left\{-\frac{\beta a_{i} k_{i}}{2}\left|\mathbf{x}_{i}-\mathbf{X}_{i}\right|^{2}+\beta \hat{\mu}_{i} a_{i}\right\} d \mathbf{x}_{i}\right\} \\
& =\frac{1}{|D|+e^{\beta \hat{\mu}_{i}} Z_{i}}\left(e^{\beta \hat{\mu}_{i}} \int_{D} \mathbf{x}_{i} \exp \left\{-\frac{\beta k_{i}}{2}\left|\mathbf{x}_{i}-\mathbf{X}_{i}\right|^{2}\right\} d \mathbf{x}_{i}\right) .
\end{aligned}
$$

A.4. Mean Potential. The expectation value of the mean potential $\hat{V}_{\text {DMD }}$ is obtained as follows:

$$
\begin{aligned}
\mathbb{E}^{\hat{\nu}}\left[\hat{V}_{\mathrm{DMD}}\right] & =\sum_{j=1}^{N} \mathbb{E}^{\hat{\nu}}\left[\frac{a_{j} k_{j}}{2}\left|\mathbf{x}_{j}-\mathbf{X}_{j}\right|^{2}\right] \\
& =\sum_{j=1}^{N} \frac{\Pi_{k \neq j}^{N}\left\{|D|+e^{\beta \hat{\mu}_{k}} Z_{k}\right\}}{\hat{Z}} e^{\beta \hat{\mu}_{j}} \int_{D} \frac{k_{j}}{2}\left|\mathbf{x}_{j}-\mathbf{X}_{j}\right|^{2} \exp \left\{-\frac{\beta k_{j}}{2}\left|\mathbf{x}_{j}-\mathbf{X}_{j}\right|^{2}\right\} d \mathbf{x}_{j} \\
& =\sum_{j=1}^{N} \frac{c_{j} k_{j}}{2 Z_{j}} \int_{D}\left|\mathbf{x}_{j}-\mathbf{X}_{j}\right|^{2} \exp \left\{-\frac{\beta k_{j}}{2}\left|\mathbf{x}_{j}-\mathbf{X}_{j}\right|^{2}\right\} d \mathbf{x}_{j} .
\end{aligned}
$$

A.5. Free Energy Gradients. The DMD free energy gradient, with respect to $c_{i}$, is computed as follows

$$
\begin{aligned}
\frac{\partial F}{\partial c_{i}} & =-\beta^{-1} \frac{1}{Z} \frac{\partial Z}{\partial c_{i}}+\frac{\partial \boldsymbol{\mu}}{\partial c_{i}} \cdot \mathbf{c}+\mu_{i} \\
& =-\beta^{-1} \frac{1}{Z}\left\{\sum \int \beta \frac{\partial \boldsymbol{\mu}}{\partial c_{i}} \cdot \mathbf{a} \exp \left\{-\beta V_{\mathrm{DMD}}+\boldsymbol{\mu} \cdot \mathbf{a}\right\} d \mathbf{x}\right\}+\frac{\partial \boldsymbol{\mu}}{\partial c_{i}} \cdot \mathbf{c}+\mu_{i} \\
& =-\frac{\partial \boldsymbol{\mu}}{\partial c_{i}} \cdot \mathbb{E}^{\nu}[\mathbf{a}]+\frac{\partial \boldsymbol{\mu}}{\partial c_{i}} \cdot \mathbf{c}+\mu_{i}=\mu_{i}
\end{aligned}
$$

This is (3.18). The gradient of $\hat{F}$ with respect to any of the parameters defining $\hat{\nu}$ vanishes at the minimizer of $\mathcal{R}$. To see this, recall that since we have a minimizer of $\mathcal{R}$,

$$
\frac{\partial \mathcal{R}(\hat{\nu} \| \nu)}{\partial p}=0
$$

for any parameter, $p$, such as $k_{i}$ and $\mathbf{X}_{i}$. Since we can write

$$
\beta^{-1} \mathcal{R}=\hat{F}-F
$$


and $F$ does not depend on the parameters, when a derivative is taken with respect to $c_{i}$, chain rule terms involving the parameters vanish at the minimizers. Therefore,

$$
\begin{aligned}
\frac{\partial \hat{F}}{\partial c_{i}}= & -\beta^{-1} \frac{1}{\hat{Z}} \frac{\partial \hat{Z}}{\partial c_{i}}+\frac{\partial \hat{\boldsymbol{\mu}}}{\partial c_{i}} \cdot \mathbf{c}+\hat{\mu}_{i}+\frac{\partial \mathbb{E}^{\hat{\nu}}[\Delta V]}{\partial c_{i}} \\
= & \hat{\mu}_{i}+\frac{1}{\hat{Z}} \sum_{a_{i}} \int \Delta V \beta\left(\partial_{c_{i}} \hat{\boldsymbol{\mu}} \cdot \mathbf{a}\right) \exp \{-\beta \hat{V}+\beta \hat{\boldsymbol{\mu}} \cdot \mathbf{a}\} \\
& -\frac{1}{Z} \mathbb{E}^{\hat{\nu}}[\Delta V]\left(\sum_{a_{i}} \int \beta\left(\partial_{c_{i}} \hat{\boldsymbol{\mu}} \cdot \mathbf{a}\right) \exp \{-\beta \hat{V}+\beta \hat{\boldsymbol{\mu}} \cdot \mathbf{a}\}\right),
\end{aligned}
$$

and this gives us (3.19).

\section{Appendix B. Weak Compactness of the set of Measures}

Lemma 1. The set $\mathcal{A}_{D, K}$ is weakly compact, in the sense that if $\left\{\gamma_{n}\right\}$ is any sequence in $\mathcal{A}_{D, K}$, it has a weakly converging subsequence in $\mathcal{A}_{D, K}$.

Proof. Given any sequence of $\gamma_{n} \in \mathcal{A}_{D, K}$, we have sequences $\left(\mathbf{X}^{(n)}, \mathbf{k}^{(n)}\right) \in$ $\bar{D}^{N} \times[0, K]^{N}$. Since the set is compact, it has a convergent subsequence,

$$
\mathbf{X}_{i}^{\left(n_{m}\right)} \rightarrow \mathbf{X}_{i}^{(\star)}, \quad k_{i}^{\left(n_{m}\right)} \rightarrow k_{i}^{(\star)}
$$

Let $\gamma_{\star}$ be the measure associated with $\mathbf{X}^{(\star)}$ and $\mathbf{k}^{(\star)}$. Clearly, $\gamma_{\star} \in \mathcal{A}_{D, K}$.

Given any bounded continuous function $f$ on the set $X$, we will now show

$$
\mathbb{E}^{\gamma_{n m}}[f] \rightarrow \mathbb{E}^{\gamma_{\star}}[f] \text {. }
$$

From (2.16), so long as the $c_{i} \in(0,1) Z_{n}=Z_{\star}$. For brevity, let

$$
\begin{gathered}
\hat{V}_{\mathrm{DMD}}\left(\mathbf{x}, \mathbf{a} ; \mathbf{X}^{\left(n_{m}\right)}, \mathbf{k}^{\left(n_{m}\right)}\right)=\hat{V}^{(m)}(\mathbf{x}, \mathbf{a}) \rightarrow \hat{V}^{(\star)}(\mathbf{x}, \mathbf{a}) \\
\hat{\boldsymbol{\mu}}\left(\mathbf{X}^{\left(n_{m}\right)}, \mathbf{k}^{\left(n_{m}\right)}\right)=\hat{\boldsymbol{\mu}}^{(m)} \rightarrow \hat{\boldsymbol{\mu}}^{(\star)}
\end{gathered}
$$

Therefore, when we take differences,

$$
\begin{aligned}
& \left|\mathbb{E}^{\gamma_{n_{m}}}[f]-\mathbb{E}^{\gamma_{\star}}[f]\right| \\
& \leq \frac{1}{Z_{\star}} \sum_{\mathbf{a}} \int_{D^{N}}|f(\mathbf{x}, \mathbf{a})|\left|e^{-\beta \hat{V}^{(m)}(\mathbf{x}, \mathbf{a})+\beta \hat{\boldsymbol{\mu}}^{(m)} \cdot \mathbf{a}}-e^{-\beta \hat{V}^{(\star)}(\mathbf{x}, \mathbf{a})+\beta \hat{\boldsymbol{\mu}}^{(\star)} \cdot \mathbf{a}}\right| \\
& \leq \frac{1}{Z_{\star}} \sum_{\mathbf{a}} \int_{D^{N}}|f(\mathbf{x}, \mathbf{a})| e^{-\beta \hat{V}^{(\star)}(\mathbf{x}, \mathbf{a})+\beta \hat{\boldsymbol{\mu}}^{(\star)} \cdot \mathbf{a}}\left|e^{-\beta\left(\hat{V}^{(m)}(\mathbf{x}, \mathbf{a})-\hat{V}^{(\star)}(\mathbf{x}, \mathbf{a})\right)+\beta\left(\hat{\boldsymbol{\mu}}^{(m)}-\boldsymbol{\mu}^{(\star)}\right) \cdot \mathbf{a}}-1\right|
\end{aligned}
$$

Since $\mathbf{x}$ and $\mathbf{a}$ are elements of bounded sets, and

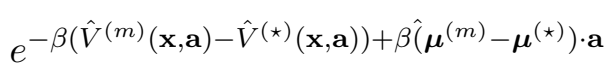

depends continuously upon $\mathbf{X}^{(m)}$ and $\mathbf{k}^{(m)}$, we then have there exists some constant such that for all $\mathbf{x} \in \bar{D}$ and $\mathbf{a} \in\{0,1\}$,

$$
\left|e^{-\beta\left(\hat{V}^{(m)}(\mathbf{x}, \mathbf{a})-\hat{V}^{(\star)}(\mathbf{x}, \mathbf{a})\right)+\beta\left(\boldsymbol{\mu}^{(m)}-\boldsymbol{\mu}^{(\star)}\right) \cdot \mathbf{a}}-1\right| \leq C\left(\left|\mathbf{X}^{(m)}-\mathbf{X}^{(\star)}\right|+\left|\mathbf{k}^{(m)}-\mathbf{k}^{(\star)}\right|\right)
$$


Therefore,

$$
\left|\mathbb{E}^{\gamma_{n_{m}}}[f]-\mathbb{E}^{\gamma_{\star}}[f]\right| \leq C\left(\left|\mathbf{X}^{(m)}-\mathbf{X}^{(\star)}\right|+\left|\mathbf{k}^{(m)}-\mathbf{k}^{(\star)}\right|\right) \mathbb{E}^{\gamma_{\star}}[|f|]
$$

and we have weak convergence.

\section{REFERENCES}

[1] F. F. Abraham, J. Q. Broughton, N. Bernstein, and E. Kaxiras. Spanning the length scales in dynamic simulation. Computers In Physics, 12(6):538-546, 1998.

[2] X. Blanc, C. Le Bris, and F. Legoll. Analysis of a prototypical multiscale method coupling atomistic and continuum mechanics. M2AN. MathematicalModelling and Numerical Analysis, 39(4):797-826, 2005.

[3] A. Chaimovich and M.S. Shell. Relative entropy as a universal metric for multiscale errors. Physical Review E, 81(6):060104, June 2010.

[4] E. Dontsova, J. Rottler, and C.W. Sinclair. Solute-defect interactions in Al-Mg alloys from diffusive variational Gaussian calculations. Physical Review B, 90(17):174102, November 2014.

[5] D.A. Drew and S.L. Passman. Theory of multicomponent fluids. Springer New York, 1999.

[6] P. Dupuis and R.S. Ellis. A weak convergence approach to the theory of large deviations. Wiley Series in Probability and Statistics: Probability and Statistics. John Wiley \& Sons Inc., New York, 1997. A Wiley-Interscience Publication.

[7] Wolfgang Eckhardt, Alexander Heinecke, Reinhold Bader, Matthias Brehm, Nicolay Hammer, Herbert Huber, Hans-Georg Kleinhenz, Jadran Vrabec, Hans Hasse, Martin Horsch, Martin Bernreuther, ColinW. Glass, Christoph Niethammer, Arndt Bode, and Hans-Joachim Bungartz. 591 TFLOPS multi-trillion particles simulation on SuperMUC. In JulianMartin Kunkel, Thomas Ludwig, and HansWerner Meuer, editors, Supercomputing, volume 7905 of Lecture Notes in Computer Science, pages 1-12. Springer Berlin Heidelberg, 2013.

[8] A.L. Gibbs and F.E. Su. On Choosing and Bounding Probability Metrics. International Statistical Review, 70(3):419-435, December 2002.

[9] G. Henkelman and H. Jónsson. A dimer method for finding saddle points on high dimensional potential surfaces using only first derivatives. J. Chem. Phys., 111(15):7010-7022, 1999.

[10] M.A. Katsoulakis and P. Plecháč. Information-theoretic tools for parametrized coarsegraining of non-equilibrium extended systems. The Journal of Chemical Physics, 139(7):074115, 2013.

[11] S.Y. Kim, D. Perez, and A.F. Voter. Local hyperdynamics. J. Chem. Phys., 139(14):144110, 2013.

[12] W.K. Kim, M. Luskin, D. Perez, E. Tadmor, and A.F. Voter. Hyper-QC: An accelerated finite-temperature quasicontinuum method using hyperdynamics. Journal of the Mechanics and Physics of Solids, 63:94-112, 2014.

[13] C. Le Bris, T. Lelièvre, M. Luskin, and D. Perez. A mathematical formalization of the parallel replica dynamics. Monte Carlo Meth. Appl., 18(2):119-146, 2012.

[14] R. LeSar, R. Najafabadi, and D.J. Srolovitz. Finite-temperature defect properties from free-energy minimization. Physical Review Letters, 63(6):624, 1989.

[15] J. Li, S. Sarkar, W.T. Cox, T.J. Lenosky, E. Bitzek, and Y. Wang. Diffusive molecular dynamics and its application to nanoindentation and sintering. Physical Review B, 84(5):054103, August 2011.

[16] M. Luskin and C. Ortner. Atomistic-to-continuum coupling. Acta Numerica, 22:397508, 2013. 
[17] A. Majda, R. Kleeman, and D. Cai. A mathematical framework for quantifying predictability through relative entropy. Methods and Applications of Analysis, 9(3):425444, 2002.

[18] R. Najafabadi, D.J. Srolovitz, and R. LeSar. Finite temperature structure and thermodynamics of the Au S5 (001) twist boundary. Journal of Materials Research, 5(11):2663, 1990.

[19] R. Najafabadi, H.Y. Wang, D.J. Srolovitz, and R. LeSar. A new method for the simulation of alloys; application to interfacial segregation. Acta Metallurgica, 39:12:30713082, 1991.

[20] F.J. Pinski, G. Simpson, A.M. Stuart, and H. Weber. Kullback-Leibler Approximation for Probability Measures on Infinite Dimensional Spaces. arXiv.org, October 2013.

[21] F.J. Pinski, G. Simpson, A.M. Stuart, and H. Weber. Algorithms for Kullback-Leibler Approximation of Probability Measures in Infinite Dimensions. arXiv.org, August 2014.

[22] R.E. Rudd and J.Q. Broughton. Coarse-grained molecular dynamics: Nonlinear finite elements and finite temperature. Physical Review B, 72:144104, 2005.

[23] S. Sarkar. Extending the Time Scale in Atomistic Simulations: The Diffusive Molecular Dynamics Method. PhD thesis, Ohio State University, 2011.

[24] S. Sarkar, J. Li, W.T. Cox, E. Bitzek, T.J. Lenosky, and Y. Wang. Finding activation pathway of coupled displacive-diffusional defect processes in atomistics: Dislocation climb in fcc copper. Physical Review B, 86(1):014115, July 2012.

[25] M.S. Shell. The relative entropy is fundamental to multiscale and inverse thermodynamic problems. The Journal of Chemical Physics, 129(14):144108, 2008.

[26] L. E. Shilkrot, W. A. Curtin, and R. E. Miller. A coupled atomistic/continuum model of defects in solids. Journal of the Mechanics and Physics of Solids, 50:2085-2106, 2002.

[27] G. Simpson and M. Luskin. Numerical analysis of parallel replica dynamics. ESAIM: Mathematical Modelling and Numerical Analysis, 47(5):1287-1314, July 2013.

[28] E.B. Tadmor, M. Ortiz, and R. Phillips. Quasicontinuum analysis of defects in solids. Philosophical Magazine A, 73(6):1529-1563, 1996

[29] G. Venturini, K. Wang, I. Romero, M.P. Ariza, and M. Ortiz. Atomistic long-term simulation of heat and mass transport. Journal Of The Mechanics And Physics Of Solids, 73(C):242-268, December 2014.

[30] Vincent A. Voelz, Gregory R. Bowman, Kyle Beauchamp, and Vijay S. Pande. Molecular simulation of ab initio protein folding for a millisecond folder NTL9 (1-39). Journal of the American Chemical Society, 132(5):1526-1528, 2010. PMID: 20070076.

[31] A.F Voter. Hyperdynamics: Accelerated molecular dynamics of infrequent events. Phys. Rev. Lett., 78(20):3908-3911, Jan 1997.

[32] A.F. Voter. Parallel replica method for dynamics of infrequent events. Phys. Rev. B, 57(22):13985-13988, Jan 1998.

[33] G. J. Wagner and W. K. Liu. Coupling of atomistic and continuum simulations using a bridging scale decomposition. Journal of Computational Physics, 190(1):249-274, 2003.

[34] L. Xu and G. Henkelman. Adaptive kinetic Monte Carlo for first-principles accelerated dynamics. J. Chem. Phys., 129(11), 2008.

E-mail address: simpson@math.drexel.edu

Department of Mathematics, Drexel University

E-mail address: luskin@umn.edu

School of Mathematics, University of Minnesota

E-mail address: srol@seas.upenn.edu 
Department of Materials Science and Engineering, Department of Mechanical Engineering and Applied Mechanics, University of Pennsylvania 